\title{
Fortuitous Discovery of Left Ventricular Apical Thrombus and Sub-Acute Lower Limb Ischemia Revealing a Hyper- Hyperhomocysteinemia in a Young Women
}

\author{
Jihad Raoui MD ${ }^{1 *}$, Bouthayna Mesmoudi MD², Soufiane Lamdak ${ }^{1}$, Gueladio Koné ${ }^{1}$, Elizabeth \\ O. Ugoani MD ${ }^{3}$, Fellat Rokiya ${ }^{4}$ \\ ${ }^{I}$ Resident doctor, Cardiology A Department, Ibn Sina Teaching Hospital, Rabat, Morocco \\ ${ }^{2}$ Resident doctor, Cardiology Center in the Mohammed V Military Instruction Hospital of Rabat, Morocco \\ ${ }^{3}$ Resident doctor, Cardiology B Department, Ibn Sina Teaching Hospital, Rabat, Morocco \\ ${ }^{4}$ Assistant Head of Department Cardiology B, IbnSina Teaching Hospital, Rabat, Morocco
}

*Corresponding Author: Jihad Raoui MD, Resident doctor, Cardiology A Department, Ibn Sina Teaching Hospital, Rabat, Morocco. E-mail: raoui.jihad@gmail.com

\begin{abstract}
Introduction: Hyperhomocysteinemia is a biological marker that could be identified in the venous thrombotic events and rarely during acute arterial thrombotic events. The consequences can be serious. Effective diagnostic strategy is needed to optimize the management.

Case Report: We report the case of a 29-year-old patient who presented with typical infarctoid pain and notion of intermittent claudication of the lower limbs, in whom clinical examination found subacute ischemia of the lower limbs. The trans-thoracic echocardiography show an apex akinesia with voluminous apical thrombus, an arterial doppler confirming an arterial obliterant arteropathy of the lower limbs.
\end{abstract}

The test for thrombophilia showed an hyperhomocysteinemia, the patient was operated in emergency with thrombectomy whose anatomopathological study confirmed this diagnosis.

Surgical treatment and anticoagulation was associated with vitamins and trace elements supplementation. Once treatment established, the patient did not present a recurrent thrombotic episode.

Conclusion: Major hyperhomocysteinemia seems to be associated with an increased risk of acute arterial thrombosis. This marker might be considered in nutritional deficiency situations with appropriate support on the vascular, metabolic and nutrition level.

Keywords: Hyperhomocysteinemia, Arterial thrombosis, sub-acute lower limb ischemia

\section{INTRODUCTION}

Hyperhomocysteinemia is characterized by an abnormally high concentration of homocysteine (a sulfurated amino acid produced through the methionine metabolism).

Hyperhomocysteinemia is classified as mild to moderate $(15-30 \mu \mathrm{mol} / \mathrm{L})$, intermediate (>31$100 \mu \mathrm{mol} / \mathrm{L})$, and severe (>100 $\mu \mathrm{mol} / \mathrm{L})$ [1]. It has been shown that patients with moderate hyperhomocysteinemia are characterized by a prothrombotic, and dysfirinolytic state and homocysteine level is an independent predictor of thrombotic events. [2]

Hyperhomocysteinemia (HHcy) might play a causative role in the development of coronary artery disease (CAD) and cardiovascular (CV) disease and originates from an interplay of environmental factors.

\section{OBSERVATION}

We report the case of a 29-year-old patient with no significant cardiovascular risk factor or history, who presents 3 months before hospitalization with dyspnea stage II-III of NYHA months associated with anginal chest pain in exertion with asthma, anorexia and the notion of intermittent claudication of the lower limbs, all of this evolving in a context of alteration of the general state.

On the day she was admitted to the emergency room, she had retrosternal chest pain with burn 
type with lower limb pain, the cardiac examination was normal but the vascular examination found subacute ischemia of the two lower limbs.

At the EKG: sinus tachycardia with QS aspect in septo-apical. The trans-thoracic echocardiography show an apex akinesia which was the seat of a voluminous mass arriving at the middle segments of the left ventricle, measured $38 \times 25 \mathrm{~mm}$, with a fixed implant base suggestive of either apical thrombus or tumor mass.

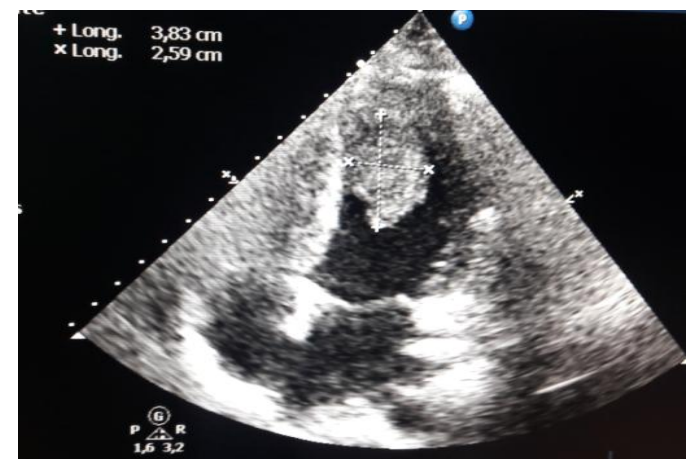

Figure1: Echocardiographic image showing apical thrombus measured $38 \times 25 \mathrm{~mm}$

Cardiac MRI showed the same echocardiography data with high suspicion of apical thrombus image.

Arterial doppler confirmed the obliterant arteropathy of the lower limbs.

As part of the etiological assessment of his thromboembolic disease a test for thrombophilia was carried out objecting to hyperhomoc ystéinémie $(100 \mu \mathrm{mol} / \mathrm{L})$

The patient was operated in an emergency with thrombectomy whose anatomopathological study confirmed this diagnosis and then put on anticoagulant and antiplatelet agent associated with vitamins and trace elements supplementation. Once treatment established, the patient did not present a recurrent thrombotic episode.

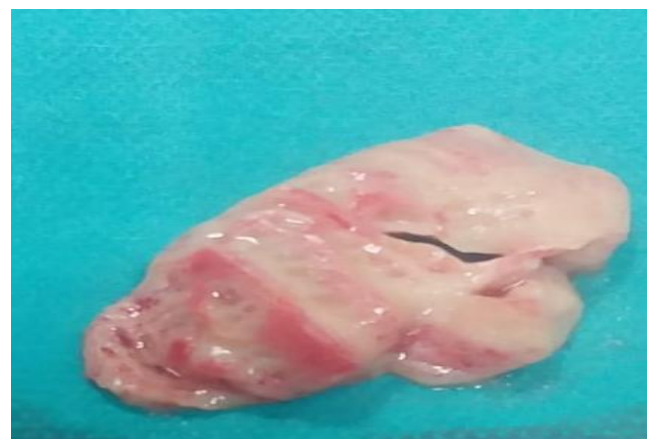

Figure2: Image of the operating part of the apical mass whose thrombotic nature is confirmed by the anatomopathological study

\section{DISCUSSION}

Homocysteine is a multipotent molecule exerting various modes of injury to the endothelium. It facilitates oxidative arterial injury, damages the vascular matrix, and induces vascular smooth muscle proliferation. [3] Moreover, homocysteine alters the thrombotic properties of the endothelium by inhibiting the expression of thrombomodulin, activating protein $\mathrm{C}$, enhancing the activity of factors 12 and 5, and augmenting platelet adhesion to the endothelial cells. [4]

The interference of homocysteine with the coagulation system creates a prothrombotic milieu. Virchow's postulates for thrombogenesis require abnormalities of blood flow, vessel wall, and blood constituents. High homocysteine fulfills the two criteria of Virchow's triad by its endothelial toxic and prothrombotic properties. Therefore, one can postulate that in conditions associated with abnormal blood flow such as stasis, high levels of homocysteine enhance thrombus formation.

Homocysteine is different than conventional hemostatic factors because it is neither a direct contributor to the coagulation pathway such as coagulation factors nor a byproduct of the thrombotic (such as fibrinopeptide $\mathrm{A}$ and prothrombin fragment 1.2) nor fibrinolytic (such as D-dimer) systems.

More than eighty epidemiological studies, including more than ten prospective studies, have shown that a moderate increase in homocysteine plasma concentration is a risk factor for cardiovascular mortality, ischemic heart disease, ischemic cerebrovascular pathology, peripheral arteropathy and also venous thrombosis [5]. Significance persists after adjustment for other risk factors, showing that it is an independent factor. Increased risk would be dose-dependent for homocysteine concentrations greater than $10 \mu \mathrm{mol} / \mathrm{l}$. It is therefore difficult to define a target homocysteinemia value at present. This is a risk factor whose power is still limited.

The Physician's Health Study [6] of 14,916 men with no known vascular involvement, followed for 5 years, showed that a $12 \%$ increase in homocysteinemia tripled the risk of myocardial infarction after adjustment for other coronary risk factors.

Boushey et al.'s meta-analysis. [7] included 4,000 patients from 38 studies published up to 
Fortuitous Discovery of Left Ventricular Apical Thrombus and Sub-Acute Lower Limb Ischemia Revealing a Hyper- Hyperhomocysteinemia in a Young Women

June 1994 on the vascular risk of hyperhomocysteinemia. The odds-ratio for coronary, cerebrovascular or peripheral arterial disease was estimated at 1.6 for men and 1.8 for women. Ten percent of the coronary risk in the general population would be attributable to homocysteine. It is suggested that an increase of $5 \mu \mathrm{mol} / \mathrm{l}$ in total plasma homocysteine increases coronary risk as well as an increase in total cholesterol of $0.5 \mathrm{mmol} / \mathrm{l}$. This shows that it is a risk factor whose power is nevertheless limited

Numerous studies have focused on the effect of vitamin treatment on the plasma concentration of homocysteine. They are all consistent, showing the effectiveness of these therapies even in the absence of nutritional deficiency. In a recent meta-analysis [8], a daily treatment with $0.5 \mathrm{mg}$ folic acid decreased homocysteinemia by $25 \%$, allowing normalization in the majority of cases. The addition of vitamin B12 orally at the dose of $0.5 \mathrm{mg}$ per day resulted in an additional $7 \%$ reduction. However, the addition of vitamine B6 did not provide any further improvement. If, to date, there is no evidence of effective therapeutic management of hyperhomocystein- emia, it is important to note that two randomised trials are currently underway to demonstrate the effect of folate treatment on cardiovascular morbimortality. [8-9]

Finally, it should be noted that the literature has not shown that correcting hyperhomocysteinemia decreases the rate of recurrence of thrombotic events [10-11] for stroke, myocardial infarction, cardiovascular mortality, arteriovenous fistula thrombosis or venous thrombosis. The authors of this review and meta-analysis agree on the existence of an association between hyperhomocysteinemia and thrombotic event without causality found. [12].

\section{CONCLusion}

High plasma homocysteine levels are a risk factor for deep-vein thrombosis in the general population and rarely arteriel thrombosis.

Hyper-homocysteinemia is an established risk factor for atherosclerosis and vascular disease.

\section{REFERENCES}

[1] Weiss N, Keller C, Hoffmann U, Loscalzo J. Endothelial dysfunction and atherothrombosis in mild hyperhomocysteinemia. Vasc Med. 2002; 7(3):227-39.

[2] Bienvenu T, Ankri A, Chadefaux B, et al. Elevated total plasma homocysteine, a risk factor for thrombosis. Relation to coagulation and firinolytic parameters. Thromb Res. 1993; 70:123-129. http://dx.doi.org/10.1016/0049-38 48(93)90153-F

[3] Ling Q, Hajjar KA. Inhibition of endothelial cell thromboresistance by homocysteine. J Nutr. 2000; 130(suppl 2S):373S-376S.

[4] Dardik R, Varon D, Tamarin I, Zivelin A, Salomon O, Shenkman B, Savion N. Homocysteine and oxidized low density lipoprotein enhanced platelet adhesion to endothelial cells under flow conditions: distinct mec

[5] Welch G.N., Loscalzo J. Homocysteine and at herothrombosis. N Eng J Med 1998; 338:104 2-50.

[6] Refsum H., Ueland M., Nygaard O. et coll. Homocysteine and cardiovase

[7] Rosenberg I.H., Bostom A., Jacques P. et coll. Gene-diet interactions in plasma homocysteine regulation. Cur Op Endocrinol Diabetes 1996 ; 3 : 497-500

[8] Stampfer M.J., Malinow M.R., Willett W.C. et coll. A prospective study of plasma homocysteine and risk of myocardial infarction in US physicians. JAMA 1992; 268: 877-81.

[9] Boushey C.J., Beresford S.A., Omenn G.S. et coll. A quantitative assessment of plasma homocysteine as a risk factor for vascular disease. JAMA $1995 ; 274: 1049-57$

[10] Clarke R. and homocysteine lowering trialists. Lowering blood homocysteine with folic acid based supplements: meta-analysis of randomized trials. Br Med J 1998 ; 316 : 894-8

[11] Gatt A, Makris M. Hyperhomocysteinemia and venous thrombosis. Semin Hematol 2007;44:70-6.

[12] Jardine MJ, Kang A, Zoungas S, Navaneethan $\mathrm{SD}$, Ninomiya T, Nigwekar SU, et al. The effect of folic acid based homocysteine lowering on cardiovascular events in people with kidney disease: systematic review and meta-analysis. BMJ 2012; 344 e3533.

Citation: Jihad Raoui MD, et al. Fortuitous Discovery of Left Ventricular Apical Thrombus and Sub-Acute Lower Limb Ischemia Revealing a Hyper-Hyperhomocysteinemia in a Young Women, ARC Journal of Cardiology. 2020; 6(1): 10-12. doi: dx.doi.org/10.20431/2455-5991.0601003.

Copyright: (1) 2020 Authors. This is an open-access article distributed under the terms of the Creative Commons Attribution License, which permits unrestricted use, distribution, and reproduction in any medium, provided the original author and source are credited. 